\title{
Planar Hall effect sensor for magnetic micro- and nanobead detection
}

Ejsing, Louise Wellendorph; Hansen, Mikkel Fougt; Menon, Aric Kumaran; Ferreira, H.A.; Graham, D.L.; Freitas, P.P.

Published in:

Applied Physics Letters

Link to article, DOI:

$10.1063 / 1.1759380$

Publication date:

2004

Document Version

Publisher's PDF, also known as Version of record

Link back to DTU Orbit

Citation (APA):

Ejsing, L. W., Hansen, M. F., Menon, A. K., Ferreira, H. A., Graham, D. L., \& Freitas, P. P. (2004). Planar Hall effect sensor for magnetic micro- and nanobead detection. Applied Physics Letters, 84(23), 4729-4731.

https://doi.org/10.1063/1.1759380

\section{General rights}

Copyright and moral rights for the publications made accessible in the public portal are retained by the authors and/or other copyright owners and it is a condition of accessing publications that users recognise and abide by the legal requirements associated with these rights.

- Users may download and print one copy of any publication from the public portal for the purpose of private study or research.

- You may not further distribute the material or use it for any profit-making activity or commercial gain

- You may freely distribute the URL identifying the publication in the public portal

If you believe that this document breaches copyright please contact us providing details, and we will remove access to the work immediately and investigate your claim 


\title{
Planar Hall effect sensor for magnetic micro- and nanobead detection
}

\author{
L. Ejsing, ${ }^{\text {a) }}$ M. F. Hansen, and A. K. Menon \\ Department of Micro and Nanotechnology (MIC), Technical University of Denmark (DTU), \\ Building 345 East, DK-2800 Kongens Lyngby, Denmark
}

\author{
H. A. Ferreira, D. L. Graham, and P. P. Freitas \\ Institute of Engineering of Systems and Computers-Microsystems and Nanotechnologies (INESC-MN), \\ Rua Alves Redol 9, Lisbon 1000-029, Portugal
}

(Received 26 January 2004; accepted 8 April 2004; published online 20 May 2004)

\begin{abstract}
Magnetic bead sensors based on the planar Hall effect in thin films of exchange-biased permalloy have been fabricated and characterized. Typical sensitivities are $3 \mu \mathrm{V} / \mathrm{Oe} \mathrm{mA}$. The sensor response to an applied magnetic field has been measured without and with coatings of commercially available $2 \mu \mathrm{m}$ and $250 \mathrm{~nm}$ magnetic beads used for bioapplications (Micromer-M and Nanomag-D, Micromod, Germany). Detection of both types of beads and single bead detection of $2 \mu \mathrm{m}$ beads is demonstrated, i.e., the technique is feasible for magnetic biosensors. Single $2 \mu \mathrm{m}$ beads yield 300 $\mathrm{nV}$ signals at $10 \mathrm{~mA}$ and 15 Oe applied field. (C) 2004 American Institute of Physics.
\end{abstract}

[DOI: $10.1063 / 1.1759380]$

Detection of magnetic beads for bioapplications has been carried out with giant magnetoresistance (GMR) sensors, ${ }^{1-3}$ spin valve sensors, ${ }^{4-7}$ and a silicon Hall sensor. ${ }^{8}$ Planar Hall sensors have been used to detect nanotesla magnetic fields. ${ }^{9,10}$ This letter demonstrates that an exchangebiased permalloy planar Hall sensor can be used to detect micro- and nanomagnetic beads used for bioapplications.

Formerly, a planar Hall sensor made of nickel was demonstrated capable of detecting superparamagnetic microbeads. ${ }^{11}$ Here, we report on the use of permalloy as a sensing material since it has a higher anisotropic magnetoresistance (AMR) effect than nickel. To control the anisotropy and to achieve a well-defined single domain initial magnetization state, the permalloy layer is exchange coupled to a MnIr antiferromagnetic layer. The $200 \AA$ thick exchangecoupled permalloy layer has an increased effective anisotropy field, $H_{\mathrm{an}} \approx 59 \mathrm{Oe}$, defining the sensor saturation field.

The planar Hall effect is based on the AMR of ferromagnetic materials. The transverse voltage on a planar Hall cross depends on the orientation of the magnetization of the material with respect to the longitudinal current running through the material. The electrical output signal in the planar Hall geometry (inset of Fig. 1) is ${ }^{12}$

$$
V_{y}=\frac{\left(\rho_{\|}-\rho_{\perp}\right) I_{x}}{t} \sin (\phi) \cos (\phi),
$$

where $t$ is the metal layer thickness, $I_{x}$ is the applied current, and $\phi$ is the angle between the current and the in-plane magnetization vector, M. $\rho_{\|}$and $\rho_{\perp}$ are the resistivities when the magnetization is parallel and perpendicular to the current, respectively. $\Delta \rho=\left(\rho_{\|}-\rho_{\perp}\right)$ is responsible for AMR and the planar Hall effect. For a $200 \AA$ thick permalloy film, the resistivity variation is approximately $2.2 \% .^{13}$

A schematic drawing of the planar Hall sensor is presented in the inset of Fig. 1. Initially, the magnetization lies along the easy axis, which is also the direction of the applied

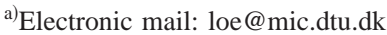

current, $I_{x}$. When a magnetic field, $H_{y}$, is applied in the $y$ direction, the magnetization rotates by an angle $\phi$ in the sensor plane. This changes the electrical output signal by the amount $V_{y}$. For small angles, the signal can be expressed as

$$
V_{y}=\frac{\left(\rho_{\|}-\rho_{\perp}\right) l_{x}}{t} \frac{H_{y}}{H_{\mathrm{an}}} .
$$

Therefore, in small applied fields compared to the anisotropy field, the response of the sensor is linear. For bead detection, $H_{y}$ in expression (2) represents the applied external field plus the sum of the $y$ components of the field created by the homogeneously magnetized spherical beads weighed by the bead-to-sensor area fraction.

A micrograph of the cleanroom fabricated planar Hall sensor is shown in Fig. 1. The sensor layer structure Ta (30 $\AA) / \mathrm{NiFe}(50 \AA) / \mathrm{MnIr}(200 \AA) / \mathrm{NiFe}(200 \AA) / \mathrm{Ta}(30 \AA)$ (see

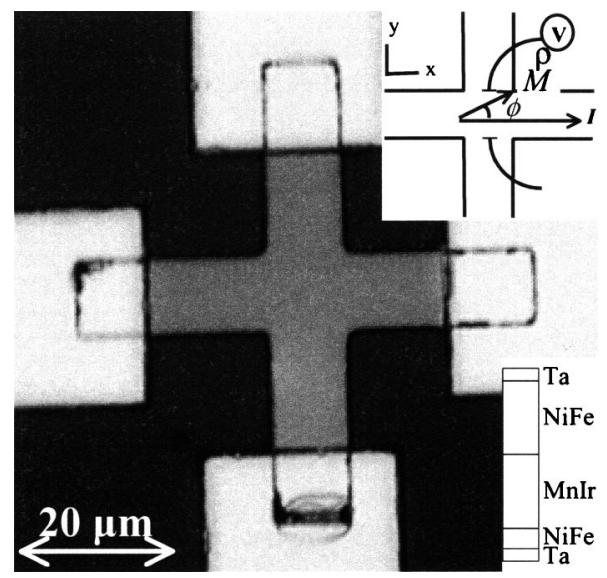

FIG. 1. Micrograph of the planar Hall sensor. The cross is made of exchange-biased permalloy, and the central area is the $10 \mu \mathrm{m} \times 10 \mu \mathrm{m}$ sensitive area of the sensor. Current leads are made of $0.3 \mu \mathrm{m}$ thick Al. The top inset shows the planar Hall sensor geometry. The magnetic field to be detected is applied in the detection plane, along the $y$ direction. In this plane, the current is applied in the $x$ direction and the voltage, $V_{y}$, is measured in the $y$ direction. The bottom inset illustrates the cross-sectional layer structure of the sensor. 
TABLE I. Physical properties of Micromod-M beads and Nanomag-D beads. ${ }^{a}$

\begin{tabular}{lllll}
\hline \hline & Diameter & Concentration & \multicolumn{1}{c}{ Density } & \multicolumn{1}{c}{$\chi^{\mathrm{b}}$} \\
\hline Micromer-M & $2 \mu \mathrm{m}$ & $>25 \mathrm{mg} / \mathrm{ml}$ & $1.4 \mathrm{~g} / \mathrm{cm}^{3}$ & $0.3 \pm 0.1(\mathrm{SI})$ \\
Nanomag-D & $250 \mathrm{~nm}$ & $>10 \mathrm{mg} / \mathrm{ml}$ & $4.0 \mathrm{~g} / \mathrm{cm}^{3}$ & $6 \pm 2(\mathrm{SI})$ \\
\hline \hline
\end{tabular}

${ }^{\mathrm{a} S e e}$ Ref. 14.

${ }^{b}$ Measured at Institute of Engineering of Systems and Computers-Microsystems and Nanotechnologies (INESC-MN).

cross-sectional diagram in the inset of Fig. 1) was prepared by ion-beam deposition on a passivated $3 \mathrm{in}$. Si wafer, then covered by sputtered antireflecting TiWN (150 A). Ion-beam deposition conditions are given in Gehanno et al. ${ }^{13}$ Here, MnIr stands for $\mathrm{Mn}_{76} \mathrm{Ir}_{24}$ and $\mathrm{NiFe}$ stands for $\mathrm{Ni}_{80} \mathrm{Fe}_{20}$. During ion-beam metal deposition, a magnetic field of $40 \mathrm{Oe}$ was applied in order to form an easy axis in the ferromagnetic NiFe layers. Sensors (crosses of $10 \mu \mathrm{m} \times 10 \mu \mathrm{m}$ ) were patterned by direct write laser lithography and ion-beam milling. Al current leads ( $0.3 \mu \mathrm{m}$ thick) were sputtered after a soft etch of the contact surface and defined by photolithography and lift off. The sensor structure was then passivated by a sputtered $\mathrm{SiO}_{2}(2000 \AA)$ layer and contact pads were opened by reactive ion etching.

The active sensing layer is the $\mathrm{NiFe}(200 \AA)$ layer. For characterization of the planar Hall sensors, electromagnetic coils were used to provide a tuneable magnetic field and the sensor response signal was measured as a function of applied field strength. Direct currents of 1, 5, and $10 \mathrm{~mA}$ are applied and direct voltage drops are measured using a voltmeter; no electronic noise reduction is used. A typical sensitivity is $S$ $=3 \mu \mathrm{V} / \mathrm{Oe} \mathrm{mA}$ in the range of $\pm 15 \mathrm{Oe}$, where the signal is linear within $2.8 \%$. This sensitivity is reproducible for a specific sensor and for sensors on the same chip.

For demonstration of bead detection, a droplet containing superparamagnetic Micromod beads $(2 \mu \mathrm{m}$ or $250 \mathrm{~nm}$ diameter, ${ }^{14}$ physical properties are summarized in Table I) was placed on top of the sensor while measuring the voltage drop. The beads were magnetized with a -15 Oe in-plane applied field generated by electromagnetic coils leading to estimated local dipole fields just below the beads of $+1.5 \mathrm{Oe}$ for $2 \mu \mathrm{m}$ beads, and +30 Oe for $250 \mathrm{~nm}$ beads.

Figure 2 presents the bead detection results. Current through the sensor is $5 \mathrm{~mA}$ and the applied field is -15 Oe. At time $t=100 \mathrm{~s}$, the $2 \mu \mathrm{m}$ beads are added onto the chip

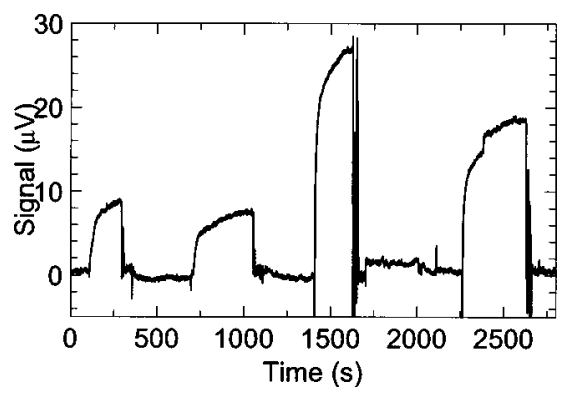

FIG. 2. Bead detection measurements. A constant voltage $\left(V_{0}=\right.$ $-464 \mu \mathrm{V}$ ) is subtracted from the signal to give a baseline at $0 \mu \mathrm{V}$. At times $t=100 \mathrm{~s}$ and $t=700 \mathrm{~s}$ the $2 \mu \mathrm{m}$ beads are added to the chip, and at times $t=300 \mathrm{~s}$ and $t=1050 \mathrm{~s}$ they are washed away. The same is done for $250 \mathrm{~nm}$ beads (added at $t=1400 \mathrm{~s}$ and $t=2250 \mathrm{~s}$, removed at $t=1650 \mathrm{~s}$ and $t$ $=2650 \mathrm{~s}$ ). The signal rises when beads settle on top of the sensor and

returns to the baseline when the beads are removed from the sensor. $\quad V_{\text {bead }} \sim 0.3 \mu \mathrm{V}$.
Downloaded 03 Apr 2010 to 192.38.67.112. Redistribution subject to AlP license or c and the signal rises as the beads settle on the sensor. Then, at time $t=300 \mathrm{~s}$, the beads are washed away and the signal returns to the baseline. At times $t=700 \mathrm{~s}$ and $t=1050 \mathrm{~s}$, respectively, the experiment is repeated yielding the same result. At time $t=1400 \mathrm{~s}$, the $250 \mathrm{~nm}$ beads are added to the chip and at time $t=1650 \mathrm{~s}$ the beads are washed away. The experiment is repeated with the $250 \mathrm{~nm}$ beads at times $t$ $=2250 \mathrm{~s}$ and $t=2650 \mathrm{~s}$, respectively. The $250 \mathrm{~nm}$ beads give higher saturation signals than the $2 \mu \mathrm{m}$ beads due to their higher susceptibility and higher number on top of the sensor area. Saturation of the signal occurs when the beads are piled up on top of the sensor and the addition of another bead is no longer sensed because it is too far away from the sensor to be detected.

Figure 3 shows sensor response to an increasing or decreasing number of beads over the sensing area $(2 \mu \mathrm{m}$ Micromod-M beads). Current through the sensor is $10 \mathrm{~mA}$ and applied field is -15 Oe. The estimate of how many beads are floating over the sensitive area at a given time is carried out by visual inspection through a microscope during the measurements. Specifically, the large numbers of beads

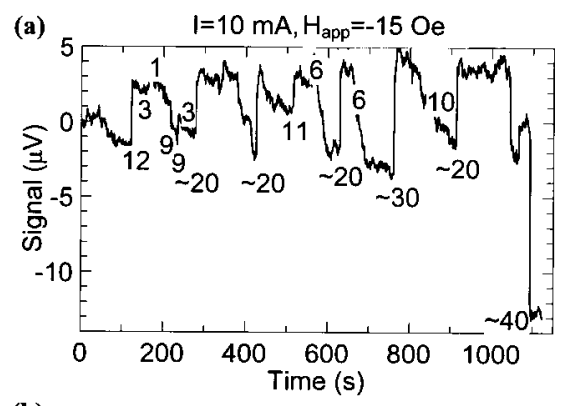

(b)

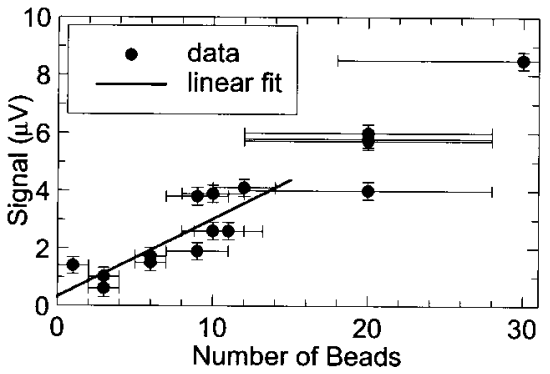

FIG. 3. Detection of clusters. The field applied with electromagnetic coils was $H_{\text {app }}=-15$ Oe. (a) The sensor signal measured while observing the sensor through an optical microscope keeping track of when beads were floating over the sensor. The numbers written on the chart are the numbers of beads entering or leaving the space over the sensitive area at the specified time. The estimate of bead number giving the voltage change at a specific time was made by visual inspection through the microscope. Specifically, the large numbers are connected with uncertainty due to the difficulty of counting beads in a limited amount of time. (b) Summary of the data from chart (a). The immediate change in signal for a given bead number entering or leaving the space over the sensor. For low bead numbers, the result is $V_{\text {bead }} \sim 0.3 \mu \mathrm{V}$. 
are connected with uncertainty since it is difficult to judge the exact number of beads constituting a cluster. For low bead numbers, the resulting one-bead signal is $V_{\text {bead }}$ $\sim 0.3 \mu \mathrm{V}$. The noise level for these dc measurements is 200-300 nV.

In conclusion, detection of commercially available $2 \mu \mathrm{m}$ and $250 \mathrm{~nm}$ superparamagnetic beads (Micromer-M and Nanomag-D) ${ }^{14}$ used for bioapplications was demonstrated using an exchange-biased permalloy planar Hall sensor. Hence, this planar Hall sensor has potential as a magnetic biosensor, e.g., for deoxyribonucleic acid detection. The technique is sufficiently sensitive to detect only a few magnetic beads and therefore also biomolecules even if only few are present in the sample. For small numbers of $2 \mu \mathrm{m}$ beads, bead detection experiments showed a single-bead signal of $V_{\text {bead }} \sim 0.3 \mu \mathrm{V}$.

Due to the cross geometry, the planar Hall sensor uses all its active surface for bead/biomolecule detection, which is not the case for meandering-type GMR or spin valve sensors, where almost one-half of the target biomolecules will sit over a nonsensing area. A second advantage, not exploited here, concerns the theoretically higher signal-to-noise $(\mathrm{S} / \mathrm{N})$ ratio for planar Hall sensors when compared with GMR or spin valve sensors. For comparable sensing areas, although the spin valve sensor yields signals 5 to 10 times higher, the planar Hall sensor should have a noise level (in the $1 / f$ dominated low-frequency regime) about 20 times lower offering the possibility of single bead detection for nanometer-sized beads.

Using a lock-in amplifier to obtain a bandwidth of $1 \mathrm{~Hz}$ will improve the $\mathrm{S} / \mathrm{N}$ considerably. For the reported sensor size with an applied current of $15 \mathrm{~mA}$ and defining the minimum detectable signal as four times the noise level, the theoretical $1 / f$ noise level is $\approx 0.6 \mathrm{nV}$ and the sensor should be able to detect a single $250 \mathrm{~nm}$ bead. If the sensor size is reduced to $2.5 \mu \mathrm{m} \times 2.5 \mu \mathrm{m}$ and the current is reduced to 10 $\mathrm{mA}$, it should be possible to detect a single $40 \mathrm{~nm}$ bead with $\chi=6(\mathrm{SI})$.

Future work includes integration with microfluidic systems along with a demonstration of the planar Hall sensor as a biosensor and single micro- and nanobead detection.

${ }^{1}$ D. R. Baselt, G. U. Lee, M. Natesan, S. W. Metzger, P. E. Sheehan, and R. J. Colton, Biosens. Bioelectron. 13, 731 (1998).

${ }^{2}$ R. L. Edelstein, C. R. Tamanaha, P. E. Sheehan, M. M. Miller, D. R. Baselt, L. J. Whitman, and R. J. Colton, Biosens. Bioelectron. 14, 805 (2000).

${ }^{3}$ M. M. Miller, P. E. Sheehan, R. L. Edelstein, C. R. Tamanaha, L. Zhong, S. Bounnak, L. J. Whitman, and R. J. Colton, J. Magn. Magn. Mater. 225, 138 (2001).

${ }^{4}$ D. L. Graham, H. Ferreira, J. Bernardo, P. P. Freitas, and J. M. S. Cabral, J. Appl. Phys. 91, 7786 (2002).

${ }^{5}$ H. A. Ferreira, D. L. Graham, P. P. Freitas, and J. M. S. Cabral, J. Appl. Phys. 93, 7281 (2003).

${ }^{6}$ D. L. Graham, H. A. Ferreira, P. P. Freitas, and J. M. S. Cabral, Biosens. Bioelectron. 18, 483 (2003)

${ }^{7}$ G. Li, V. Joshi, R. L. White, S. X. Wang, J. T. Kemp, C. Webb, R. W. Davis, and S. Sun, J. Appl. Phys. 93, 7557 (2003).

${ }^{8}$ P. A. Besse, G. Boero, M. Demierre, V. Pott, and R. Popovic, Appl. Phys. Lett. 80, 4199 (2002).

${ }^{9}$ F. Montaigne, A. Schuhl, F. N. Van Dau, and A. Encinas, Sens. Actuators 81, 324 (2000).

${ }^{10}$ F. N. Van Dau, A. Schuhl, R. J. Childress, and M. Sussiau, Sens. Actuators, A 53, 256 (1996).

${ }^{11}$ L. Ejsing, M. F. Hansen, and A. K. Menon, Proceedings of the 17th European Conference on Solid-State Transducers, Eurosensors 2003, 21-24 September 2003, Guimarães, Portugal (University of Minho, Guimarães, Portugal, 2003), p. 1095.

${ }^{12}$ R. C. O'Handley, Modern Magnetic Materials (Wiley, New York, 2000).

${ }^{13}$ V. Gehanno, P. P. Freitas, A. Veloso, J. Ferreira, B. Almeida, J. B. Sousa, A. Kling, J. C. Soares, and M. F. Silva, IEEE Trans. Magn. 35, 4361 (1999).

${ }^{14} \mathrm{http} / / / \mathrm{www} \cdot$ micromod.de 